\title{
Implementation of Wireless Communication using NFC (Near Field Communication Technology) in Mobile Computing
}

\author{
Indra Kishor ${ }^{1}$, Urvashi Tiwari ${ }^{2}$ \\ ${ }^{1,2}$ Arya Institute of Engineering \& Technology, Kukas, Jaipur, Raj. Department of Computer Science \& Engineering. \\ Rajasthan Technical University Kota
}

\begin{abstract}
The advancement in wireless technologies with years of constant progress is developing a communication. In short Communication has enhanced to convey the information quickly to the users. Wireless communication has become a very popular and widely used method for short-range data transmission between the devices like laptops, PDAs, and mobile phones. The main objective of our paper is to introduce "NFC". NFC is one of the latest wireless communication technologies. As a short-range wireless connectivity technology, NFC offers safe yet simple and intuitive communication between electronic devices. Users of NFC-enabled devices can simply point or touch their devices to other NFC-enabled elements in the environment to communicate with them, making application and data usage easy and convenient. With NFC technology, communication occurs when an NFC-compatible device is brought within a few centimeters of another NFC device or an NFC tag. The big advantage of the short transmission range is that it inhibits eavesdropping on NFC - enabled transactions. NFC technology opens up exciting new usage scenarios for mobile device.
\end{abstract}

Keywords: NFC, Wi-fi, Bluetooth Networks, Infra Red

\section{Introduction}

Mobile computing is human-computer interaction by which a computer is expected to be transported during normal usage. Mobile computing involves mobile communication, mobile hardware, and mobile software. Communication issues include ad hoc and infrastructure networks as well as communication properties, protocols, data formats and concrete technologies. Thus we introducing latest wireless communication technologies like NFC, Bluetooth, infrared which are used for communication between two devices. Mobile software deals with the characteristics and requirements of mobile applications. Mobile computing is "taking a computer and all necessary files and software out into the field." NFC, Bluetooth and Infrared - all these technologies had done its part in different period. [1]

NFC is one of the latest wireless communication technology, as a short-range wireless connectivity technology. NFC offers safe yet simple and intuitive communication between electronic devices. Near field communication (NFC) is a set of standards for Smartphone and similar devices to establish radio communication with each other by touching them together or bringing them into close proximity, usually no more than a few inches. Present and anticipated applications include contactless transactions, data exchange, and simplified setup of more complex communications such as Wi-Fi.[2]

Infrared, as the name indicates, the communication is established via infrared signals. This technology is used by most of the remote controls. This technology is device Specific and there should be direct line of sight between (max 3 feet) transmitter and receiver. This communication is more secure since it can be intercepted by a device and it's one-to-one. This technology since it is not used in mobile devices anymore. [7]
Bluetooth is a wireless LAN technology that connects devices (called gadgets) in a small area. Compared to infrared, Bluetooth devices can work together, its Omnidirectional. Current devices can transfer in the range of 30 feet. Bluetooth uses radio frequency and hence transmission is possible through walls or other objects and hence it is widely used in computers, PDA, headsets, mobile handsets etc. These devices can communicate with each other irrespective of the manufacturer since it is using standard 2.4 $\mathrm{GHz}$ frequency (ISM - Industrial Scientific and Medical devices - band).[1]

\section{Related Works}

There are numerous researches happening in the field of wireless communications which has given rise to ideas how effortlessly communication happening in devices. Mobile computing and networking have inarguably emerged among the most vibrant areas in the field of telecommunications today. Customer's requirement for immediate response to their prerequisites, targeting on access to information anywhere in the world at anytime, has increased the demand for productivity and effectiveness of mobile computing and networking. Moreover, the convergence of information communications technology and computing is creating new demands and opportunities for ubiquitous computing via wireless and mobile equipment without technological restrictions. Future mobile communication networks offer a variety of high quality applications. Furthermore, several challenges are imposed by the demanding wireless networking environment, in terms of network architecture, channel estimation, propagation and especially radio resource management. The another relevant technology in a wireless communication are Bluetooth, infrared. 


\section{International Journal of Science and Research (IJSR) \\ ISSN (Online): 2319-7064}

Index Copernicus Value (2016): 79.57 | Impact Factor (2015): 6.391

\subsection{Bluetooth}

Bluetooth is a wireless LAN technology that connects devices (called gadgets) in a small area. A Bluetooth network is called a piconet. Multiple piconet form a network called a scatternet. The Bluetooth radio layer performs functions similar to those in the Internet model's physical layer. The Bluetooth baseband layer performs functions similar to those in the Internet model's MAC sublayer. A Bluetooth frame consists of data as well as hopping and control mechanisms. A frame is one, three, or five slots in length with each slot equal to $625 \mu \mathrm{s}$. Bluetooth technology eliminates the need for numerous and inconvenient cable attachments. This technology enables users to automatically and easily connect a wide range of computing and telecommunication devices. Bluetooth makes wireless communication and networking between devices in a small localized area. In Bluetooth all the connections between devices are occurring immediately and invisible. Bluetooth is actually a standard for wireless communications between devices within a range of 10 metres. Bluetooth was officially introduced in 1998 and its intended basic purpose was to be a wire replacement technology in order to rapidly transfer voice and data. Bluetooth "Special Interest Group" is a group of companies from the telecommunications, computing and networking industries that promote the development and deployment of Bluetooth technology. Bluetooth is a high-speed, low-power microwave wireless link technology, designed to connect phones, laptops, PDAs and other portable equipments together with little or no work by the user. Unlike infra-red, Bluetooth does not require any line-of-sight positioning of connected devices like Infra-red.[1]

\subsubsection{How Bluetooth Technology Works?}

Two Bluetooth products can exchange their addresses and capabilities just after coming within the range of about 10 metres. The Bluetooth modules can be either built into electronic devices or used as an adaptor. For instance in a PC they can be built in as a PC card or externally attached via the USB port.

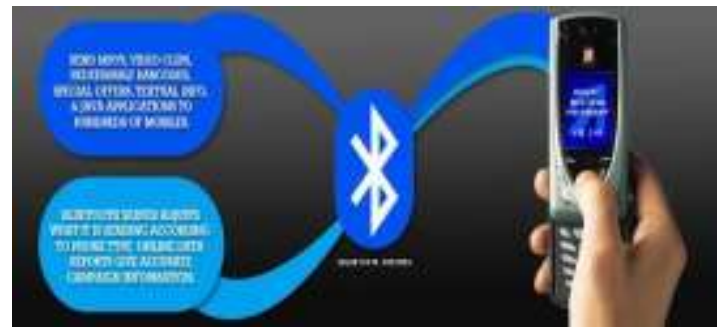

Bluetooth module Connections can be point-to-point or multipoint. The maximum range is 10 meters but can be extended to 100 meters by increasing the power. Bluetooth devices are protected from radio interference by changing their frequencies arbitrarily up to a maximum of 1600 times a second, a technique known as frequency hopping. The Bluetooth specification targets power consumption of the device from a "hold" mode consuming 30 micro amps to the active transmitting range of 8-30 milliamps which is less than $3 \%$ of the power used by a standard mobile phone. The chips also have excellent power-saving features. Automatic communication between various devices within a small area makes it possible to provide unique and innovative services to a professional worker.

\subsubsection{Piconet with Operations}

Piconet is the important factor in Bluetooth connectivity. It is simply a collection of devices connected through Bluetooth Technology. While establishing a Piconet, one unit will behave as a master and the other as slaves for the duration of Piconet connection. By installing a Bluetooth network in office you can do away with the complex task of networking between the computing devices. Each Bluetooth device could be connected to 200 other devices. It supports both point to point and point to multipoint connections. The Bluetooth technology connects all your peripherals wirelessly. Connect your pc or to printers, scanners and other devices without the trouble of cable attachments. You can increase your connections by connecting your keyboard wirelessly to your computer.

\subsubsection{Applications}

If your digital camera is Bluetooth enabled, you can send videos, images from any location to any location without the problem of connecting your cameras to the mobile phone. Connect your wireless headset to your pc to your mobile phone or mobile computer to keep your hands free for more important tasks when you are at the office or in your car.

\subsubsection{Advantages}

Bluetooth Devices are Wireless. In addition to improving safety as a result of eliminating the clutter of wires and associated hazardous connections, Bluetooth wireless technology also offers you many convenient advantages. Bluetooth Technology is Inexpensive. Bluetooth technology is cheap for companies to implement, which results in lower over-all manufacturing Costs. These savings are then passed on to you, the consumer. The end result: Bluetooth devices are relatively inexpensive. Bluetooth is Automatic. When two or more Bluetooth devices enter a range (Up to 30 feet) of one another, they automatically begin to communicate without you having to do anything. The best part is: The devices take care of the entire setup process, and you can go about your business. Low Energy Consumption, Bluetooth uses low power signals.

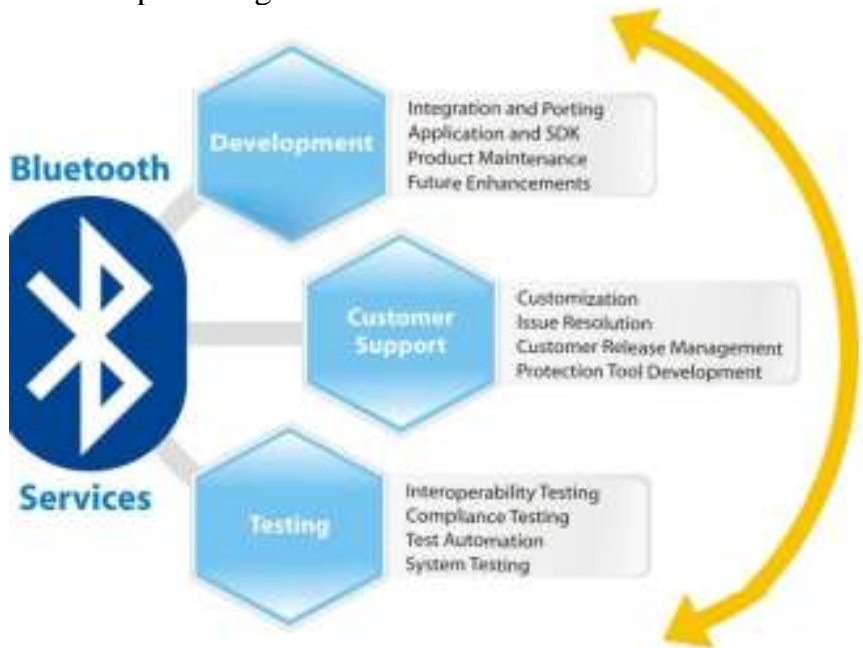

As a result, the technology requires little energy and will therefore use less battery or electrical power. Share Voice and Data. The Bluetooth standard allows compatible devices 


\section{International Journal of Science and Research (IJSR) \\ ISSN (Online): 2319-7064}

Index Copernicus Value (2016): 79.57 | Impact Factor (2015): 6.391

to share both voice and data communications. For example, it is probably no surprise that a Bluetooth enabled cell phone is capable of sharing voice communications with a compatible Bluetooth headset, however, the same cell phone may also be capable of establishing a GPRS connection to the Internet. Then, using Bluetooth, the phone can connect to a laptop. The result: The laptop is capable of surfing the web or sending and receiving email. Accessible from anywhere. Though Bluetooth has a specific range but we can access it up to very wide range and can also use it from an unanticipated range of application from mobiles to automobiles. The low power consumption, small size and low cost enables this technology to be used in the tiniest of devices. Many of us think that Bluetooth is going to be here to stay, but it is wrong. Bluetooth will be able to complete all other wireless technologies, such as Wi-Fi networking, infrared data association, etc.

\subsubsection{Disadvantage}

Bluetooth devices cannot be connected with more than one devices at a time, as it finds problem in discovering the intended recipient device. Bluetooth has a low data rate( i.e.1 MBps) in comparison to infra-red(i.e.4 MBps).

\subsection{Infrared}

Infrared represents an attractive choice for many short range applications. Its advantages include the availability of a wide bandwidth that is unregulated worldwide and that can be reused in a very dense fashion, immunity to eavesdropping, the ability to achieve very high bit rates, low signalprocessing complexity, and potentially very low cost. IR wireless communication communicates data or information in devices or systems through infrared (IR) radiation. Infrared is electromagnetic energy at a wavelength that is longer than that of red light.[7]

\subsubsection{Working}

Infrared wireless is used for short and medium-range communications and security control. For Infrared communication to work, the systems mostly operate in lineof-sight mode which means that there must be no obstruction between the transmitter (source) and receiver (destination). Infrared is used in television remote controls and security systems. In the electromagnetic spectrum, infrared radiation lies between microwaves and visible light, therefore, they can be used as a source of communication.[1]

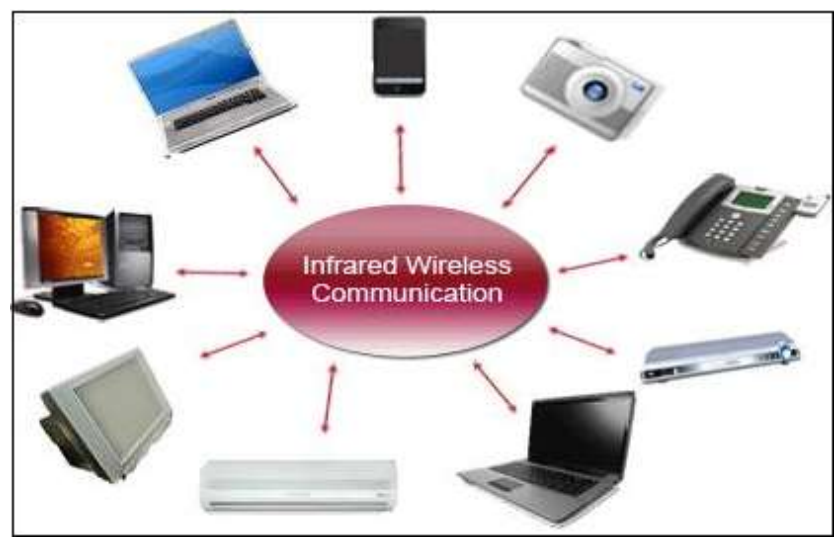

Working of Infrared Wireless Communication
A photo LED transmitter and a photodiode receptor are required for successful IR communication. The LED transmitter transmits the infrared signal in the form of nonvisible light, which is captured and retrieved as information by the photo receptor. In this way, the information between the source and the target is transferred.

The source and/or destination can be laptops, mobile phones, televisions, security systems and any other device that supports wireless communication.

Infrared emitters and detectors capable of high-speed operation are available at low cost. The infrared spectral region offers a virtually unlimited bandwidth that is unregulated worldwide. Infrared and visible light are close together in wavelength, and they exhibit qualitatively similar behavior. Both are absorbed by dark objects, diffusely reflected by light-colored objects, and directionally reflected from shiny surfaces. Both types of light penetrate through glass, but not through walls or other opaque barriers, so that infrared transmissions are confined to the room in which they originate. This signal confinement makes it easy to secure transmissions against casual eavesdropping, and it prevents interference between links operating in different rooms. Thus, infrared wireless LAN's can potentially achieve a very high aggregate capacity, and their design. Infrared may be simplified, since transmissions in different rooms need not be coordinated. When an infrared link employs intensity modulation with direct detection (IM/DD), the short carrier wavelength and large-area, square-law detector lead to efficient spatial diversity that prevents multipath fading. By contrast, radio links are typically subject to large fluctuations in received signal magnitude and phase.

Freedom from multipath fading greatly simplifies the design of infrared links. The infrared medium is not without drawbacks, however. Because infrared cannot penetrate walls, communication from one room to another requires the installation of infrared access points that are interconnected via a wired backbone. In many indoor environments there exists intense ambient infrared noise, arising from sunlight, incandescent lighting and fluorescent lighting, which induce noise in an infrared receiver.

\section{Proposed Work}

To enhance the world in a modern technologies, we need to reduce a working time and making a communication very fast, so we have to build our wireless communication more reliable by using communication channels like NFC by replacing previous technology Bluetooth and infrared. In short Communication has enhanced to convey the information quickly to the users. As wireless communication has become a very popular and widely used method for shortrange data transmission between mobile devices like laptops, PDAs, and mobile phones.

\subsection{Near Field Communication (NFC)}

Near Field Communication (NFC) is a technology for contactless short-range communication. Based on the Radio

\section{Volume 6 Issue 12, December 2017}




\section{International Journal of Science and Research (IJSR) \\ ISSN (Online): 2319-7064}

Index Copernicus Value (2016): 79.57 | Impact Factor (2015): 6.391

Frequency Identification (RFID), it uses magnetic field induction to enable communication between electronic devices. The number of short-range applications for NFC technology is growing continuously, appearing in all areas of life. Especially the use in conjunction with mobile phones offers great opportunities. One of the main goals of NFC technology has been to make the benefits of short-range contactless communications available to consumers globally.

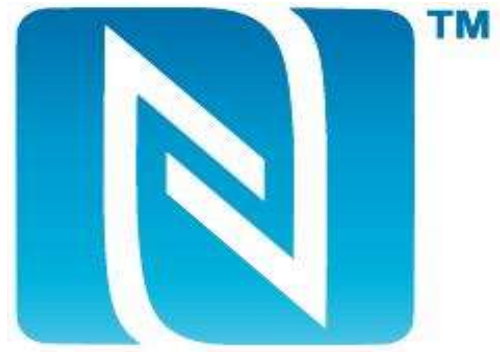

NFC is a set of short-range wireless technologies, typically requiring a distance of $10 \mathrm{~cm}$ or less.[2] NFC operates at 13.56 $\mathrm{MHz}$ on ISO/IEC 18000-3 air interface and at rates ranging from $106 \mathrm{Kbit} / \mathrm{s}$ to $424 \mathrm{Kbit} / \mathrm{s}$. NFC always involves an initiator and a target; the initiator actively generates an RF field that can power a passive target. This enables NFC targets to take very simple form factors such as tags, stickers, key fobs, or cards that do not require batteries. The existing radio frequency (RF) technology base has so far been driven by various business needs, such as logistics and item tracking. While the technology behind NFC is found in existing applications, there has been a shift in focus most notably, in how the technology is used and what it offers to consumers. With just a point or a touch, NFC enables effortless use of the devices and gadgets we use daily. Here are some examples of what a user can do with an NFC mobile phone in an NFC-enabled environment:

- Download music or video from smart poster.

- Exchange business card with another phone.

- Print an image on printer.

Use a point-of-sale terminal to pay for a purchase, the same way as with a standard contactless credit card.

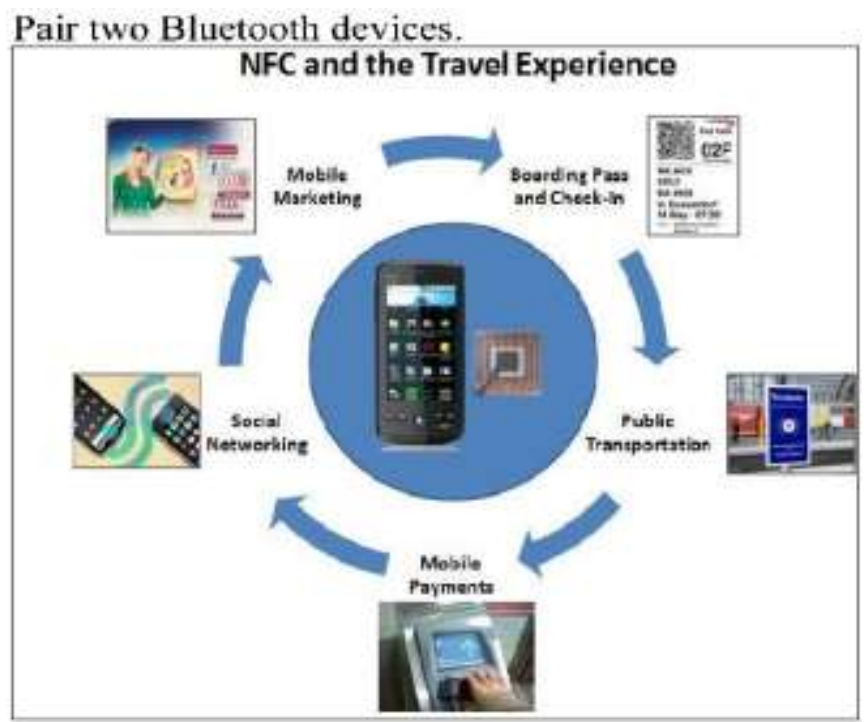

An NFC-enabled phone functions much like standard contactless smart cards that are used worldwide in credit cards and in tickets for public transit systems. Once an application, such as a credit card application, has been securely provisioned to the NFC-enabled phone, the customer can pay by simply waving the phone at a point-ofsale reader. The NFC phone also offers enhanced security, enabling the user to protect the secure applications through the phone's user interface features.[2]

\subsubsection{NFC Devices}

Like ISO 14443, NFC communicates via magnetic field induction, where two loop antennas are located within each other's near field, effectively forming an air-core transformer. It operates within the globally available and Unlicensed radio frequency ISM band of $13.56 \mathrm{MHz}$, with a bandwidth of almost $2 \mathrm{MHz}$ Working distance with compact standard antennas: up to $20 \mathrm{~cm}$.

Supported data rates: 106, 212, or $424 \mathrm{kbit} / \mathrm{s}$. There are two modes:

- Passive Communication Mode: The Initiator device provides a carrier field and the target device answers by modulating existing field. In this mode, the Target device may draw its operating power from the Initiator-provided electromagnetic field, thus making the Target device a transponder.

- Active Communication Mode: Both Initiator and Target device communicate by alternately generating their own field. A device deactivates its RF field while it is waiting for data. In this mode, both devices typically need to have a power supply.

NFC devices are able to receive and transmit data at the same time. Thus, they can check the radio frequency field and detect a collision if the received signal does not match with the transmitted signal. NFC builds upon Radio-Frequency Identification (RFID) and contactless smartcard technologies that enable stored data to be actively "read" at a distance. RFID is a powerful enabling technology that is being applied in an astonishing range of applications and uses, from supply chain management and product inventory control to identity authentication and access control. However, as RFID technologies become widely deployed, the possibility of unwanted identification, tracking and surveillance may increase, as may the likelihood of data interception, "cloning" and misuse. NFC is particularly well-suited for use in mobile devices, where its operation and behaviour are controlled by the device owners. NFC has three principal modes of operation:

\section{Reader/ writer}

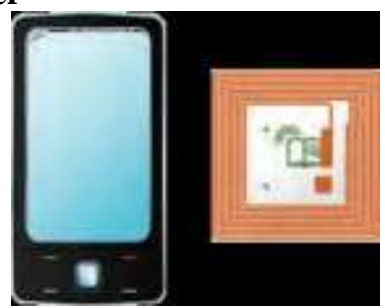




\section{International Journal of Science and Research (IJSR) \\ ISSN (Online): 2319-7064}

Index Copernicus Value (2016): 79.57 | Impact Factor (2015): 6.391

This NFC mode enables mobile devices to read data stored in passive RFID tags embedded in public posters, displays, and products - and to act upon that data that contains a Uniform Resource Locator (URL), which is an Internet encoding of access instructions for a file or Web address, or instruction for making a call, or the SMS instruction for sending a text message. This NFC mode also enables mobile devices to write data to some tags - notably virtual tags in other devices.

\section{Card Emulation}

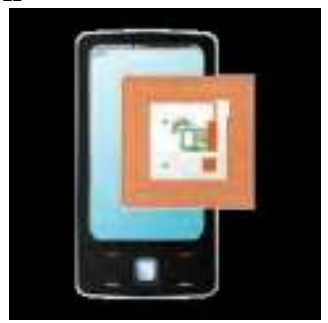

This NFC mode enables mobile device owners to make a contactless business transaction, in the same way smart cards are used today. This mode of operation enables mobile devices to be used for identification, payment and access control applications.

\section{Peer}

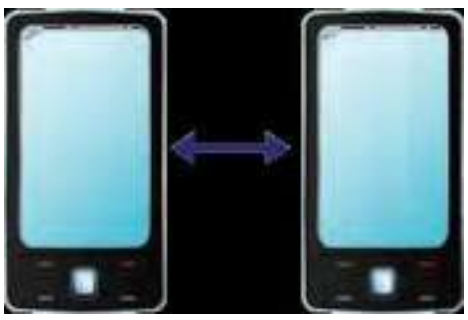

This NFC mode enables mobile devices to more easily interact with each other (i.e., each phone has to be equipped with NFC and the enabling applications) to quickly launch a mobile communications bearer for sharing data with each other, whether to exchange business cards, photos, documents or other type of personal information in "peer-topeer" data transfers.[7]

Mobile devices that allow for system-to-system data transfers, or pairing of devices to enable interaction, may trigger privacy concerns, including the following:

- Unwanted data leakage or collection.

- Tracking of a user's location.

- Identifying users in situations where they wish to remain anonymous.

- Improper redirection to an unknown website. Initiation of an unknown service.

- Receipt of unwanted content.

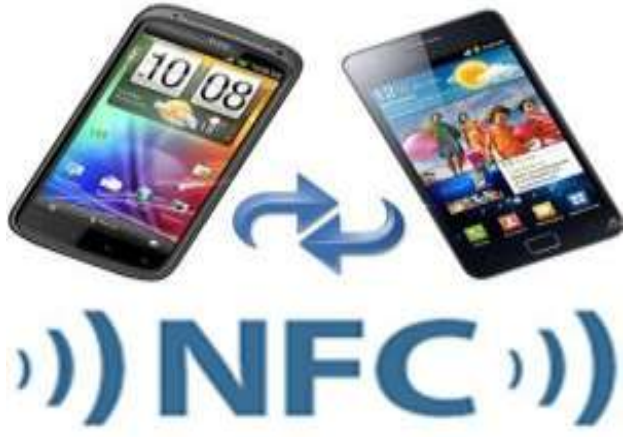

3.1.2 The NFC privacy concerns are-

1)NFC requires less than 4 centimeters of close proximity for interactions. At this close range, users will have foreknowledge of the person or device that they are interacting with.

2)NFC interactions are based on a "tapping" consumer gesture, where both NFC devices either touch or are within a few centimeters of each other in order to initiate an NFC interaction. This makes "skimming" and "eavesdropping" very difficult.

3)NFC capabilities should be disabled when the screen or keyboard of an NFC-enabled mobile phone is locked. Additionally, the mobile platform should permit users to disable the NFC function, as well as any alternative communication technology (e.g., Bluetooth or Wi-Fi) used for peer-to-peer transfer of personal information. This prevents unintended NFC interactions.

4)NFC implementations should provide user feedback on interaction requests from another NFC-enabled device. This reduces hidden or unwanted NFC interactions.

5)NFC-initiated sharing of personal data should be accomplished with use of regenerated identifiers, to avoid association of a device and its user with an NFC interaction

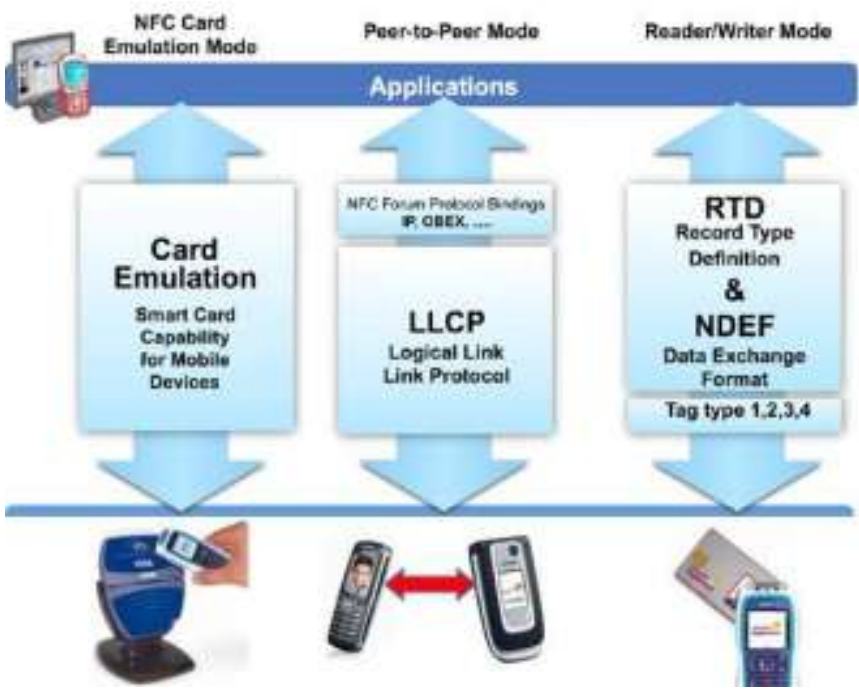

The NFC technology supports extension mechanisms for transfer of large amounts of data, the current radio frequency allocation to NFC requires close proximity of NFC devices during interactions, which creates a user experience that is not conducive to long data transfers. This is why NFC is typically expected to be used for either small data transfer interactions or for launching larger data transfers with an alternative mobile wireless communication technology, such

\section{Volume 6 Issue 12, December 2017}

\section{www.ijsr.net}

Licensed Under Creative Commons Attribution CC BY 


\section{International Journal of Science and Research (IJSR) \\ ISSN (Online): 2319-7064}

Index Copernicus Value (2016): 79.57 | Impact Factor (2015): 6.391

as Bluetooth, WiFi and mobile data service.[2]

NFC provides a capability for initiating wireless communication interactions. There are four main reference use cases for these interactions:

1) Service initiation (e.g., read a tag on a poster and launch a Web browser to get product discount coupons).

2) Pairing of devices (e.g., activate a Bluetooth headset by tapping on the mobile accessory).

3) Peer-to-peer data transfer (e.g., quickly and easily transfer information between mobile devices with a simple touch).

4) Secure NFC card (e.g., mobile device acts as an access, loyalty, or payment contactless smart card).

\subsection{Future Scope}

- Electronic ticketing - airline tickets, concert/event tickets, and others

- Electronic money

- Travel cards

- Identity documents

- Mobile commerce

- Electronic keys - car keys, house/office keys, hotel room keys, etc.

- NFC can be used to configure and initiate other wireless network connections such as Bluetooth, WI-Fi or Ultrawideband.

- NFC for Health Monitoring in Daily Life

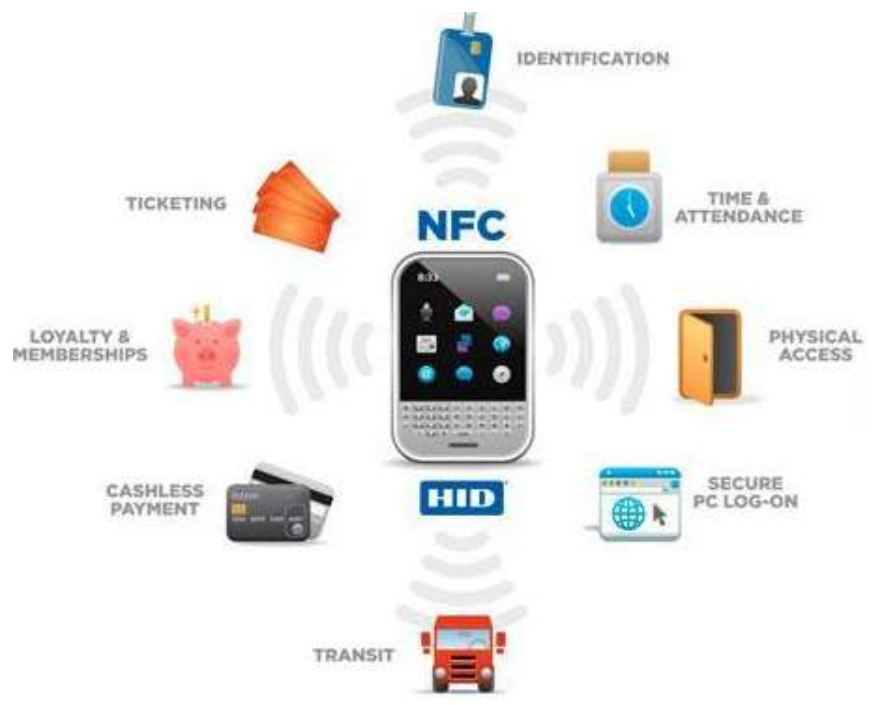

\subsubsection{Comparison with Bluetooth and Infrared}

\section{Infrared}

Compared to other short-range communication technologies, which have been integrated into mobile phones, NFC simplifies the way consumer devices interact with one another and obtains faster connections. The problem with infrared, the oldest wireless technology introduced in 1993, is the fact that a direct line of sight is required, which reacts sensitively to external influences such as light and reflecting objects. The significant advantage over bluetooth is the shorter set-uptime. Instead of performing manual configurations to identify the other's phone, the connection between two NFC devices is established at once $(<0,1 \mathrm{~s})$. Table points out these different capabilities of NFC, Bluetooth and infrared. All these protocols are point-to-point protocols. Bluetooth also supports point-to multipoint communications. With less than $10 \mathrm{~cm}, \mathrm{NFC}$ has the shortest range. This provides a degree of security and makes NFC suitable for crowded areas.

The data transfer rate of NFC (424 kbps) is slower than Bluetooth (721kbps), but faster than infrared (115 kbps). In contrast to Bluetooth and infrared NFC is compatible to RFID. This will engage the wireless interface of the two devices and configure them to link up in a peer-to-peer network. Once the device is linked up using NFC, they can continue communication using long range and faster protocols such as Bluetooth or wireless Internet (Wi-Fi).

\subsubsection{NFC Compared with Bluetooth and Infrared}

\begin{tabular}{|c|c|c|c|c|}
\hline & NFC & $\begin{array}{l}\text { Benefits of } \\
\text { NFC }\end{array}$ & Bluetooth & IrDa \\
\hline $\begin{array}{l}\text { Network } \\
\text { Type }\end{array}$ & Point-to-point & $\begin{array}{l}\text { Easy sef-up, } \\
\text { paring a bringing } \\
\text { cose }\end{array}$ & $\begin{array}{l}\text { Point-to- } \\
\text { multipoint }\end{array}$ & Point-to-point \\
\hline Range & $40.1 \mathrm{~m}$ & $\begin{array}{l}\text { Sate, sutade for } \\
\text { conded areas }\end{array}$ & $10 \mathrm{~m}$ & $1 \mathrm{~m}$ \\
\hline Speed & $\begin{array}{l}424 \text { kbps } \\
\text { (1Nbps coming) }\end{array}$ & & $721 \mathrm{kbps}$ & $115 \mathrm{kbps}$ \\
\hline Set-up time & $0.1 \mathrm{~s}$ & $\begin{array}{l}\text { Fast tansactions } \\
\text { e.g lor putelic } \\
\text { tansport }\end{array}$ & $6 s$ & $0.5 \mathrm{~s}$ \\
\hline Modes & $\begin{array}{l}\text { Active-active, } \\
\text { active-passive }\end{array}$ & $\begin{array}{l}\text { Resder mode ard } \\
\text { cand the mode. }\end{array}$ & Active-active & Active-active \\
\hline $\begin{array}{l}\text { Compatible } \\
\text { with RF ID }\end{array}$ & Yes & $\begin{array}{l}\text { Can verkwin } \\
\text { exsing } \\
\text { intrastructure }\end{array}$ & No & No \\
\hline Costs & Low & $\begin{array}{l}\text { Aflordable fox } \\
\text { most devices }\end{array}$ & Moderate & Low \\
\hline
\end{tabular}

\subsubsection{Unique features}

NFC protocol provides some features not found in other general-purpose protocols. First of all, it is a very short-range protocol. It supports communication at distances measured in centimeters. The devices have to be literally almost touched to establish the link between them. This has two important consequences:

1) The devices can rely on the protocol to be inherently secured since the devices must be placed very close to each other. It is easy to control whether the two devices communicate by simply placing them next to each other or keeping them apart.

2) The procedure of establishing the protocol is inherently familiar to people: you want something to communicate touch it. This allows for the establishment of the network connection between the devices be completely automated and happen in a transparent manner. The whole process feels then like if devices recognize each other by touch and connect to each other once touched.

3) Another important feature of this protocol is the support for the passive mode of communication. This is very important for the battery devices since they have to place conservation of the energy as the first priority.

The protocol allows such a device, like a mobile phone, to operate in a power saving mode - the passive mode of NFC communication.[2] 


\section{International Journal of Science and Research (IJSR) \\ ISSN (Online): 2319-7064 \\ Index Copernicus Value (2016): 79.57 | Impact Factor (2015): 6.391}

\subsubsection{Uses and applications}

NFC technology is currently mainly aimed at being used with mobile phones. There are three main use cases for NFC:

- Card Emulation: the NFC device behaves like an existing contactless card

- Reader Mode: the NFC device is active and read a passive RFID tag.

- P2P Mode: two NFC devices are communicating together and exchanging information.

Plenty of applications are possible, such as:

- Mobile ticketing in public transport - an extension of the existing contactless infrastructure.

- Mobile payment - the device acts as a debit/ credit payment card.

- Smart poster - the mobile phone is used to read RFID tags on outdoor billboards in order to get info on the move.

- Bluetooth pairing - in the future pairing of Bluetooth 2.1 devices with NFC support will be as easy as bringing them close together and accepting the pairing. The process of activating Bluetooth on sides, searching, waiting, pairing and authorization will be replaced by a simple "touch" of the mobile phones.

\section{Conclusion}

As portable computers and communication terminals become more powerful and are more widely deployed, the demand for high-speed wireless communication is increasing. So, the latest wireless communication technology is a Near Field Communication i.e. NFC. NFC gives high speed and fastest sending of data rather than BT and IRDA. As near field indicates, maximum distance for communication is only 4 centimeters. NFC can interact with other device with a single wave and it's faster compared to BT (needs only 1/10 seconds to establish).

It also reduces the security risk specified in case of BT and NFC needs minimal power compared to BT. The speed of NFC is only $424 \mathrm{Kbit} / \mathrm{s}$ and it can be used in combination with BT to speed up the process. NFC is not a fashionable nice-to-have technology, but actually a technology that makes people lives easier - easier to pay for goods and services, easier to use public transport, and easier to share data between devices.

The design and deployment of NFC technology offers new conveniences and benefits to users, and represents several advances in security and privacy over traditional architectures. Nokia is a leading global player in the NFC ecosystem and is working to ensure that Privacy by Design principles are embedded in their products and services, since including NFC technologies

\section{Glossary}

- BT- Bluetooth.

- IRDA, IR- Infrared.

- NFC - Near Field Communications.

\section{References}

[1] www.en.wikipedia.org/wiki/Near_Field_Communication.

[2] Near Field Communication in the real world, turning the NFC promise into profitable, everyday applications Innovision.

[3] Mobile Near Field Communications (NFC) "Tap "n Go" Keep it Secure \& Private Ann Cavoukian, Ph.D.Information and Privacy Commissioner, Ontario, Canada.

[4] NFC Forum: www.nfc-forum.org.

[5] NFC World: www.nfcworld.com.

[6] Security in Near Field Communication (NFC) Strengths and Weaknesses Ernst Haselsteiner and Klemens Breitfuß Philips SemiconductorsMikronweg 1, 8101 Gratkorn, Austria.

[7] Wireless Infrared Communications JOSEPH M. KAHN, MEMBER, IEEE, AND JOHN R. BARRY.

\section{Author Profile}

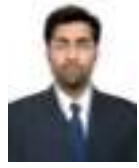

Indra Kishor Working as Assistant Professor in Arya Institute of Engg. \& Tech. Kukas Jaipur Rajasthan with M.Tech, B.Tech. degree in Computer science \& engineering from Rajasthan Technical university Kota Raj. And also three year diploma in Computer science from State board of technical university Jharkhand. Currently enhancing career in the field of education \& research.

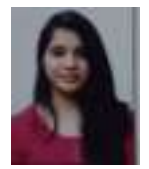

Urvashi Tiwari working as Assistant Professor in Arya Institute of Engg. And Tech. Kukas Jaipur Rajasthan, and completed M.Tech. from Banasthali university Niwai Jaipur also B.Tech from Srcem group of college, Banmore. 